\title{
Densification and thermal gradient evolution of alumina during microwave sintering at $2.45 \mathrm{GHz}$
}

Daniel Żymełka ${ }^{a}$, Sébastien Saunier ${ }^{b}$, Dominique Goeuriot ${ }^{b}$, Jérôme Molimard ${ }^{c}$

Ecole Nationale Supérieure des Mines de Saint Etienne

${ }^{a}$ Centre Microélectronique de Provence, 880 avenue de Mimet, 13541 Gardanne, France

${ }^{b}$ Science des Matériaux et des Structures, 158 Cours Fauriel, 42023 Saint Etienne, France

'Centre Ingénierie et Santé, 158 Cours Fauriel, 42023 Saint Etienne, France

\begin{abstract}
This paper presents research on the densification and thermal gradient evolution of pure and magnesia-doped alumina during microwave sintering in a multimode cavity. Dimensional changes of pellets are registered by a contactless dilatometer that is part of a specifically prepared experimental system. Measurements of dimensional changes are compared to results obtained during sintering in the conventional dilatometer and show a palpable effect of microwaves when alumina is doped by magnesia. This system also allows monitoring of temperature distribution on the surface sample from temperatures of about $700^{\circ} \mathrm{C}$. Analysis of thermal evolution shows gradient inversion for both examined powders.
\end{abstract}

Keywords: microwave sintering, alumina, dilatometer, temperature gradient 


\section{Introduction}

Microwave heating is known to be a promising sintering method, which is being explored by a growing number of researchers. The results of research on microwave sintering almost always demonstrate that time and temperatures are reduced when compared to conventional heating methods.

Xie et al. [1] compare the results on microwave and conventional sintering of pure alumina (99.97\%) in a range of temperature between 1000 and $1500^{\circ} \mathrm{C}$. A palpable enhancement (even $12 \%$ ) in density is observed for the microwave sintering method up to $1300^{\circ} \mathrm{C}$. From this temperature the differences clearly decrease, to show no difference at $1500^{\circ} \mathrm{C}$. Brosnan et al. [2] also report better results for microwave sintering of alumina. The authors indicate that thanks to microwave sintering, to obtain the same density as during conventional sintering, the temperature can be lower by up to $250^{\circ} \mathrm{C}$. Samples sintered at $1400^{\circ} \mathrm{C}$ show huge differences in density. The conventionally sintered sample was $52 \%$ dense, whereas the microwave sintered sample was around $98 \%$. However, it should be noted that the powder they used was composed of $65 \%$ of $\alpha-$ alumina and $35 \%$ of $\gamma-$ alumina, doped by $350 \mathrm{ppm} \mathrm{Y}_{2} \mathrm{O}_{3}$ and $500 \mathrm{ppm} \mathrm{MgO}$. This is important if we take into account the results presented by Fang et al. [3]. They show that the microwave effect on densification of alumina may be related to phase composition ( $\gamma$ - alumina is much more reactive than $\alpha$ - alumina). The powder with the higher reactivity leads to stronger microwave enhancement in densification. This can explain the huge difference between microwave and conventional sintering observed by Brosnan et al. [2].

Mentioned often in the literature, the reduced time of sintering for the microwave method, is mainly linked to the conversion of electromagnetic (EM) field energy into heat (rapid heating rates). Reduced sintering temperature is usually considered to have a non-thermal effect on the acceleration of mass transport. Here, both these phenomena are briefly introduced. 


\section{Microwave heating}

The efficiency of microwave heating is determined by the capability of a material to absorb microwave energy i.e. by the response of a material to an external electromagnetic field. In the case of dielectric materials, such as alumina, the magnetic field does not contribute to microwave heating. Thus for these types of materials, we consider the alternating electric field as the only external factor causing the generation of heat inside a material.

Besides the cavity parameters, the efficiency of the heating process depends on applied EM field frequency and material properties (such as the nature of the material, its purity, grain size, etc.). Depending on these parameters a time lag may occur between the field oscillations and the response of the material (its polarization). Thus the response of dielectric materials to alternating electric field oscillations is determined by the complex dielectric permittivity: $\varepsilon=\varepsilon^{\prime}+i \varepsilon^{\prime \prime}$. The first component $\varepsilon^{\prime}$ is known as a dielectric constant. The second component (the imaginary part of permittivity) is called the loss factor and accounts for all the internal loss mechanisms. The loss factor describes how efficiently a material absorbs energy, i.e. how efficiently MW energy is transformed into heat. Therefore, materials with high $\varepsilon^{\prime \prime}$ absorb microwave energy well and quickly convert it into heat.

This is why microwave heating methods permit faster heating rates than conventional heating. From an economic perspective, the reduced time of sintering using microwave methods as compared to conventional processes can be directly translated into significant energy savings and thus lower production costs.

The fast heating rate may be implemented directly when an object efficiently absorbs EM energy (materials with high $\varepsilon^{\prime \prime}$ ). Since it is known that alumina has a low $\varepsilon^{\prime \prime}$ [4], in order to initialize the absorption of microwaves, the use of heating by the so-called susceptor is required (at $2.45 \mathrm{GHz}$ ). 


\section{"Microwave effect"}

From a scientific perspective, the most interesting aspect of microwave processing is reduced sintering temperature which is called "microwave effect" and, as mentioned above, is usually considered to be a non-thermal effect of microwaves on enhanced diffusion. This effect is still not clearly identified; however researchers have proposed some hypotheses.

One of these hypotheses considers the so-called "ponderomotive effect" of the microwave EM field on enhanced ionic diffusion [5]. According to this idea, an appropriate orientation of electric field oscillations (normal to the inter-grain boundary), leads to the extraction of atoms from the intergrain boundary and facilitates the shrinkage of closed pores in ceramics [6]. This concept was developed by observations of spherical pores in zirconia ceramics. The results of these observations show the deformation (elongation and preferred orientation) of pores after microwave heating. This is not observed after using a conventional heating process. Moreover, these observations can be seen as a proof of the significance of the "ponderomotive effect" on mass transport in solids [6].

Birnboim et al. [7] studied the electric field distribution within a ceramic body on a microscopic scale. Authors considered various aspects of the fields generated in the two touching spheres model. They show a high disproportion of electric field intensity between contact zone and grain volume. The electric field in the neck zone may be many times higher than in the volume. Moreover, they indicate that, the electric field in the neck zone exhibits preferred polarization directions despite illumination by a randomly polarized wave. According to the authors, the microwaves may lead to a highly nonuniform energy deposition and accelerate mass transfer rates via ponderomotive diffusion and plasma generation.

Another concept, proposed by Raj et al. [8], in contrast to those mentioned above, assumes a local thermal effect. Authors consider a higher dielectric loss at grain boundaries than that in the grain matrix. Due to this fact, the local temperatures at the grain boundary are much higher than the average temperature of the sample, and as a result the rate of diffusional transport is enhanced. 
In the present work we study the influence of an electromagnetic field at a frequency of $2.45 \mathrm{GHz}$ on the densification of alumina, both pure and doped by magnesium oxide. The following sections present major information about materials as well as about experimental methods applied in this study. Next, the results on sintering in the multimode microwave furnace are discussed and contrasted with the conventional sintering (CS) process. In order to eliminate the influence of heating rate effects on the densification process, identical thermal profiles are applied to both methods of heating. In this work dimensional changes of the sample are collected by a homemade optical dilatometer. Moreover, this paper examines and compares the thermal gradient evolution during microwave heating of pure and doped alumina.

\section{Experimental procedure}

\subsection{Preparation of samples}

In this study we used commercial alumina powders (SM8, Baikowski), pure and doped by 800 ppm of magnesium oxide (MgO), with surface area of about $10 \mathrm{~m}^{2} / \mathrm{g}$. Both powders are uniaxially pressed at $400 \mathrm{MPa}$ and thus formed into pellets. In order to remove organic materials, after compacting, the samples are pre-heated at $600^{\circ} \mathrm{C}$ for $60 \mathrm{~min}$. Dimensions and densities of green samples are presented in Table 1. Relative green densities are calculated by a geometrical method, taking into account the theoretical density of alumina equal to $3.987 \mathrm{~g} / \mathrm{cm}^{3}$.

\begin{tabular}{c|c|c|c} 
Alumina powder & Diameter, $\mathbf{m m}$ & Height, $\mathbf{m m}$ & Relative density \\
\hline Pure, for CS & 8.12 & 5.55 & 0.57 \\
\hline Pure, for MWS & 11.995 & 3.76 & 0.568 \\
\hline Doped, for CS & 8.09 & 5.85 & 0.554 \\
\hline Doped, for MWS & 11.995 & 3.935 & 0.56
\end{tabular}

Table 1 : Dimensions and densities of green samples 


\subsection{Apparatus}

Conventional sintering is carried out in a conventional dilatometer (SETARAM "Setsys 16/18"). A multimode cavity (SAIREM) with inner dimensions of $43 \times 43 \times 49 \mathrm{~cm}^{3}$ is used for microwave sintering. The microwave generator operates at a frequency of $2.45 \mathrm{GHz}$. Forward power can be chosen between 600 and 6000 W. During heating, two different pyrometers are used for temperature measurement. The first (IRCON Modline 5G-1007) is sensitive to a wavelength of 1.6 $\mu \mathrm{m}$ in a temperature range of 250 to $1000^{\circ} \mathrm{C}$. The second pyrometer (IRCON Modline 5G-3015) is automatically activated when the temperature is equal to $1000^{\circ} \mathrm{C}$. In the same way, when the temperature is lower than $1000^{\circ} \mathrm{C}$, the first pyrometer is automatically chosen. This pyrometer is sensitive to two wavelength bands (0.75-1.05 $\mu \mathrm{m}$ and 1-1.1 $\mu \mathrm{m})$ and measures the temperature from $1000^{\circ} \mathrm{C}$. Both pyrometers were provided with "ModView" software which allows us to calculate the emissivity or so-called "emissivity slope" (e-slope) which corresponds to the ratio of the energy measured on one wavelength band to that measured on the other. The software adjusts the radiation energy measured by the pyrometer to the temperature value provided by a user, and as a result, gives an emissivity or "e-slope" value.

During the whole experiment in the microwave cavity, the sample is observed and images registered by a 12-bit CCD (Charge Coupled Device) camera (JAI RM-4200 GE) having a capture size of $2048 \mathrm{x}$ 2048 pixels. Registered images are collected for an off-line analysis. Note that the camera is not an infrared (IR) camera. This camera is sensitive to the visible and the near IR light spectrum. The pyrometers and the $C C D$ camera are presented in Figure $1 \mathrm{~A}$, just as they are during the sintering process.

\subsection{Temperature measurement and calibration procedure}

Since, in this kind of investigation, temperature measurement is crucial and highly sensitive, we pay particular attention to the problem of accurate temperature measurement. Due to the described issues with thermocouples in the microwave environment [9], the most suitable method seems to be 
pyrometry. However, in our study we use a susceptor, which greatly complicates the use of pyrometers. (The principle of temperature measurements by pyrometry is generally based on light intensity measurements. Thus any other heating source emitting a light corresponding to the wavelength of the pyrometer will affect the measurement, especially when a sample has a low emissivity or lower emissivity than the other heating source.) Undoubtedly the solution to this problem may be a temperature measurement by Raman spectroscopy [10], which allows a selective temperature measurement. Even though Raman spectroscopy is a more accurate method of measurement, pyrometry is a simple and common method and, despite its limitations, we choose to employ this system of measurement, being fully aware of potential problems.

Each pyrometer requires a rigorous calibration. In the case of a monochromatic pyrometer, an appropriate emissivity value has to be given. For a two-color pyrometer, it is necessary to establish the "emissivity slope" value. The potential emissivity changes have to be always taken into account when using a monochromatic pyrometer. In this study the monochromatic pyrometer is calibrated relative to the melting point of germanium $\left(938^{\circ} \mathrm{C}\right.$ ). A small (less than $1 \mathrm{~mm}^{3}$ ) germanium piece is placed in a shallow slot in order to prevent the germanium piece from falling off the surface sample when it is in the vertical position, see Figure 1 (B). At any heating temperature stage other than $938^{\circ} \mathrm{C}$, the emissivity changes are not verified. In this work we are interested in the part of the thermal cycle when shrinkage of alumina occurs, i.e. from about $950^{\circ} \mathrm{C}$ up to $1500^{\circ} \mathrm{C}$. Thus, a well calibrated monochromatic pyrometer allows us to accurately specify the beginning of sintering and allows us to find the "e-slope" for a second (two-color) pyrometer, i.e. we use the monochromatic pyrometer as a reference to calibrate the second pyrometer at $1000^{\circ} \mathrm{C}$. The temperature of $1000^{\circ} \mathrm{C}$ is chosen because this is the only temperature when both pyrometers cover the same temperature range. So, the "e-slope" for the second pyrometer is calculated by "ModView" software when the monochromatic pyrometer indicates $1000^{\circ} \mathrm{C}$. It is expected that for the ceramic materials "e-slope" is equal to 1 . Therefore, by comparing calculated value to expected value, (both have to be very 
close), we are able to verify if a monochromatic pyrometer is well calibrated and if the measurement during the calibration procedure is not affected by the susceptor's radiation. Here, we can indicate a high dependency of crucible design on temperature measurements, when a sample is heated in the presence of the susceptor. Figure 1 (B) represents our concept of crucible design, optimized after many trials. As we can see the access to the temperature measurements and observations is from a horizontal position. In contrast to the crucible presented in Ref. [11], this solution leads to reduced heat losses, and thus to the radiation of the susceptor. These heat losses are especially important at high temperatures and when using low loss materials (such as alumina). (When heat losses are significant and a sample does not absorb microwave energy well, then the susceptor has to produce much more heat than it does when using insulated lossy materials. In consequence the susceptor emits much more radiation, which affects the temperature measurement.) In Figure 1 (C) we see the sample as the brightest object in the optical field, thus we can assume that the radiation coming from the susceptor (which is normally very strong and reflected inside a crucible), is significantly reduced.

In these conditions, for both aluminas, the calibration procedure is performed. For monochromatic $(1.6 \mu \mathrm{m})$ pyrometer, at $938^{\circ} \mathrm{C}$, the emissivity is equal to 0.5 . When the first pyrometer indicated the temperature of $1000^{\circ} \mathrm{C}$, we calculated the "e-slope" equal to 1.001 (for a second pyrometer). Since this result is consistent with the expected value, these emissivity and "e-slope" values are used throughout this study.

One very important consideration is that the calibration procedure is performed exactly and under the same conditions as in all further experiments - during microwave heating, in the same crucible and in the presence of the susceptor. 


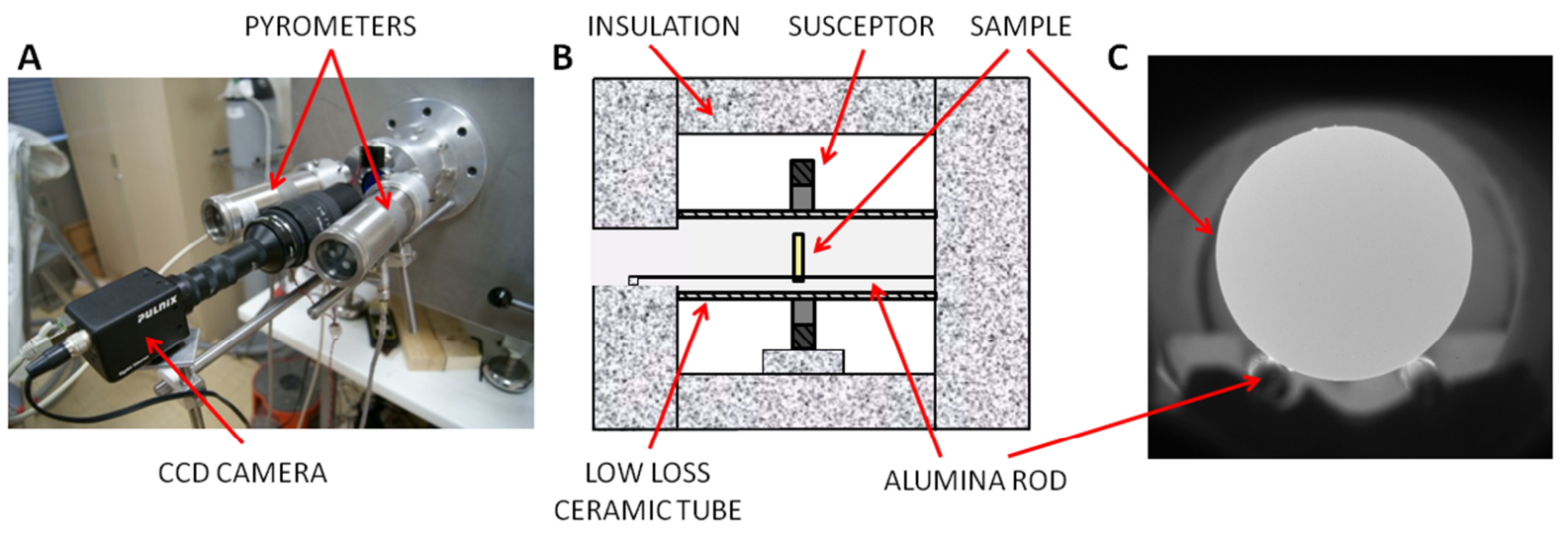

Figure 1: Microwave heating system.

\subsection{Temperature distribution and shrinkage measurements}

A dedicated method for simultaneous measurements of shrinkage and temperature distribution during microwave sintering (Ref. [11]) is applied in this work. However, one important remark should be made. As mentioned above, a specific design of the crucible is required to reduce heat losses, especially for high temperature processes. Reducing heat losses permits minimalized thermal flickering of images, and thus strongly increases the precision on dimensional variation measurements. The present system affords accuracy at least comparable to the conventional dilatometer. In the case of contactless measurement, which is not affected by the dilatation of the probe as in conventional systems, the results are even more precise. An example (registered by our system) for the microwave sintering of alumina is presented in Figure 2. As we can see the noise generated by thermal flickering is negligible. We are able to follow all dimensional changes of the sample: the shrinkage as well as the thermal dilatation. The standard deviation calculated at a temperature close to $900^{\circ} \mathrm{C}$ gives values of about 0.1 pixel, which correspond to about $1 \mu \mathrm{m}$. 


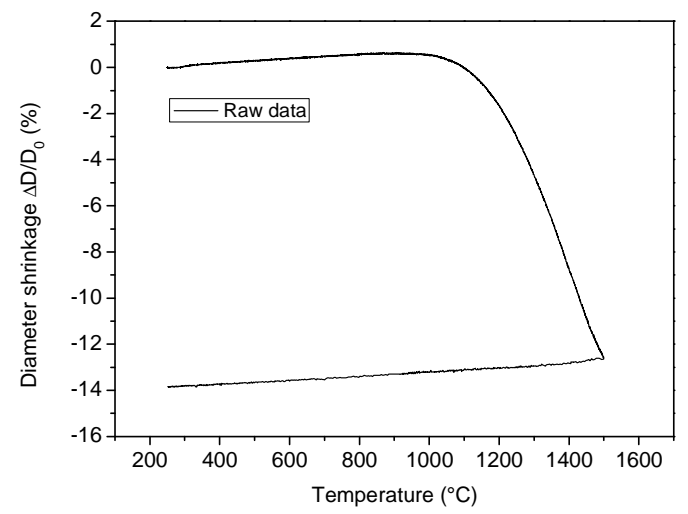

Figure 2: Dilatometric curve obtained using our optical dilatometer during microwave sintering.

\subsection{Sintering conditions}

For both sintering methods the same heating rate of $20^{\circ} \mathrm{C} / \mathrm{min}$ is applied. During conventional sintering a thermal cycle is controlled by the software provided with the dilatometer. In the case of microwave sintering, the heating rate is controlled by a homemade computer program. Figure 3 demonstrates how well the obtained thermal cycle aligns with the desired cycle. The samples are heated up to $1500^{\circ} \mathrm{C}\left(T_{\max }\right)$, but the temperature is not maintained. When using conventional sintering, after reaching $T_{\max }$, the sample is cooled down at a rate of $35^{\circ} \mathrm{C} / \mathrm{min}$. Whereas, using microwave sintering after reaching $\mathrm{T}_{\max }$, the forward power is simply turned off and the cool down temperature is not controlled. The average rate of cooling after microwave heating is calculated in the range of 1500 to $1400^{\circ} \mathrm{C}$ and is equal to $230^{\circ} \mathrm{C} / \mathrm{min}$. This is the only difference between the thermal profiles. In both cases the sintering is carried out in a non-controlled air atmosphere. 


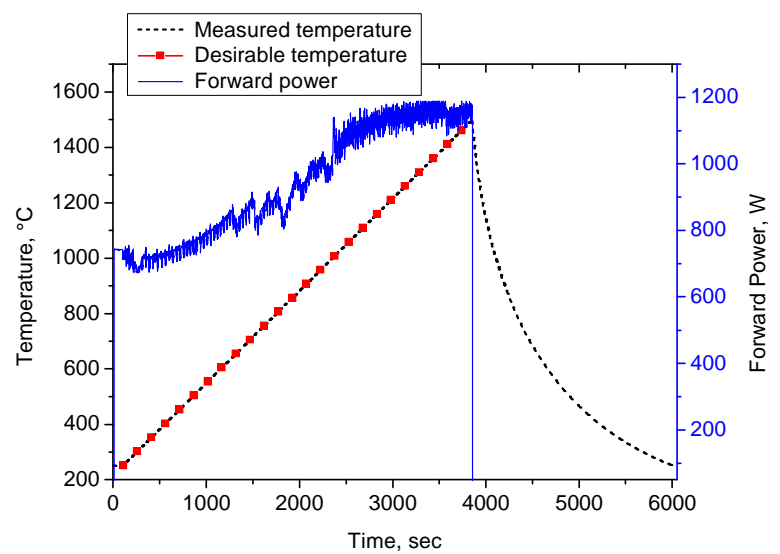

Figure 3: Example of the thermal cycle accomplished in microwave furnace controlled by a homemade computer program.

\subsection{Dilatometric characterization}

During sintering in the conventional dilatometer, the sample is in physical contact with a probe that follows the sample's dimensional changes. The problem is that during heating, due to thermal expansion, the probe changes its dimensions and affects the accuracy of measurements. To reduce this effect, the thermal expansion of the probe is calibrated by a reference experiment with a fully dense sample.

After sintering, in order to verify the isotropy of shrinkage strain, the axial and radial measurements of shrinkage are compared. The problem is that the results show anisotropy of shrinkage. Another problem is that during sintering in the conventional dilatometer, height is used to measure the shrinkage, while during microwave sintering, diameter is used to measure the shrinkage. Thus, the shrinkage curves of these two methods cannot be compared. Therefore, the results of the conventional and the microwave sintering are compared by time dependent relative density (respectively Eq. 1 and Eq. 2), calculated taking into account the anisotropic shrinkage factor " $\alpha$ " (Eq. 3). In equation 1 , the initial and the final height of the sample are expressed respectively by $h_{0}$ and $h_{f}$. The time dependent shrinkage measurement made by the conventional dilatometer is represented by $h(t)$. In equation $2, \Phi_{0}$ and $\Phi_{f}$ represent the initial and the final diameter of the 
sample, while the time dependent shrinkage $\Phi(t)$ is registered by the noncontact optical dilatometer. The final densities $\rho_{f}$ are measured by the Archimedes' method.

$$
\begin{gathered}
\rho(t)=\frac{\left(1+\alpha \cdot \frac{h_{f}-h_{0}}{h_{0}}\right)^{2}\left(1+\frac{h_{f}-h_{0}}{h_{0}}\right)}{\left(1+\alpha \cdot \frac{h(t)-h_{0}}{h_{0}}\right)^{2}\left(1+\frac{h(t)-h_{0}}{h_{0}}\right)} \cdot \rho_{f} \\
\rho(t)=\frac{\left(1+\frac{\Phi_{f}-\Phi_{0}}{\Phi_{0}}\right)^{2}\left(1+\frac{1}{\alpha} \cdot \frac{\Phi_{f}-\Phi_{0}}{\Phi_{0}}\right)}{\left(1+\frac{\Phi(t)-\Phi_{0}}{\Phi_{0}}\right)^{2}\left(1+\frac{1}{\alpha} \cdot \frac{\Phi(t)-\Phi_{0}}{\Phi_{0}}\right)} \cdot \rho_{f} \\
\alpha=\frac{\Phi_{f}-\Phi_{0}}{\Phi_{0}} \cdot \frac{h_{0}}{h_{f}-h_{0}}
\end{gathered}
$$

\section{Results}

\subsection{Dilatometric results}

Sintering analysis of pure alumina (Fig. 4) shows almost no difference up to $\mathrm{T}_{\max }$, when comparing the results obtained by both heating methods. At the maximal temperature equal to $1500^{\circ} \mathrm{C}$, calculated densities are $89 \%$ and $89.2 \%$ of theoretical density for conventional and microwave sintering respectively. Final density calculations show that during cooling in conventional dilatometer, the density of the sample increased up to $91.6 \%$, while for the MW sintered sample increased up to 89.7\%. This difference in density gain during the cooling is caused by the uncontrolled and the fast cooling rate in the microwave furnace. It shows how important is to regulate the thermal cycle, especially when making comparisons between microwave and conventional sintering processes. For this reason we only take into account the results up to $1500^{\circ} \mathrm{C}$, where thermal cycles are identical. 
The results indicate no significant influence of microwaves on density evolution for studied pure alumina.

When analyzing the density changes for alumina doped by 800ppm MgO (Fig. 5), differences between microwave and conventional sintering methods are clearly seen. For MW sintering the densification process starts earlier than for conventional heating. Density calculated at $1500^{\circ} \mathrm{C}$ is equal to $96 \%$ in contrast to $93.3 \%$ during sintering in the conventional dilatometer. The final density of the sample sintered in the MW furnace is greater (96.1\%) than the conventionally sintered sample (95.2\%).

An effect of $\mathrm{MgO}$ on densification evolution during $\mathrm{MW}$ sintering is shown in Figure 6. This figure represents differences between densities calculated for MW and conventionally sintered samples at different stages of heating. We can see that for alumina with $\mathrm{MgO}$, at a temperature around $1380^{\circ} \mathrm{C}$, the difference between both sintering methods is maximal and is equal to about $3 \%$. This difference is not spectacular, however it is sufficient to confirm the influence of microwaves on densification of alumina doped by MgO. A good complement to this study may be an analysis of alumina sintering with different concentrations of $\mathrm{MgO}$.

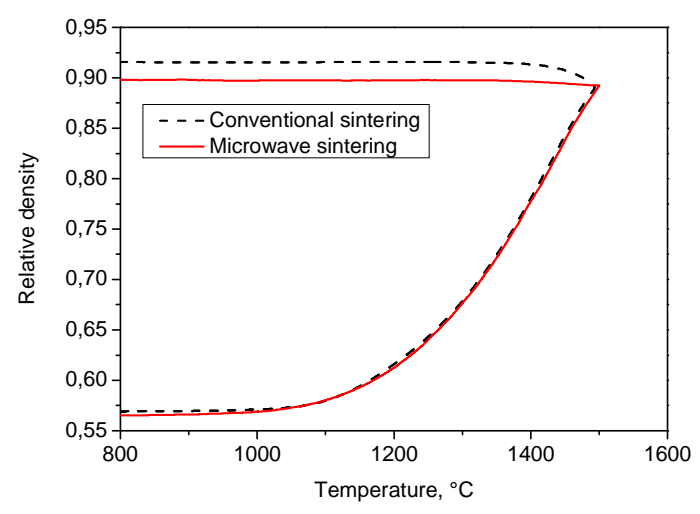

Figure 4: Comparison of relative densities for pure alumina sintered with constant heating rate $20^{\circ} \mathrm{C} / \mathrm{min}$. 


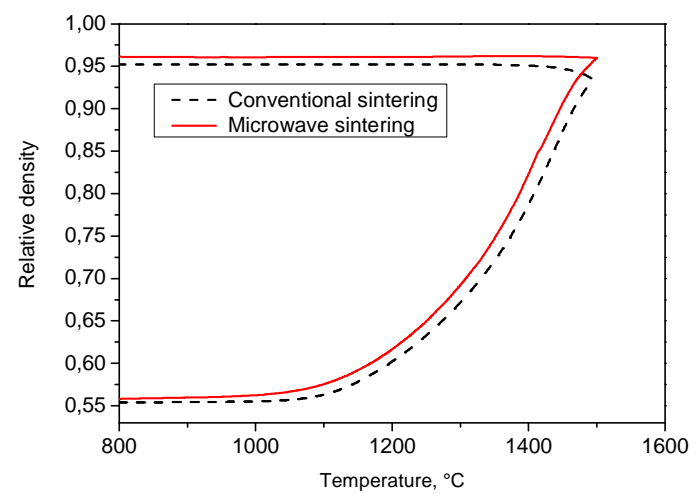

Figure 5: Comparison of relative densities for alumina doped by $800 \mathrm{ppm} \mathrm{MgO}$ and sintered with constant heating rate $20^{\circ} \mathrm{C} / \mathrm{min}$.

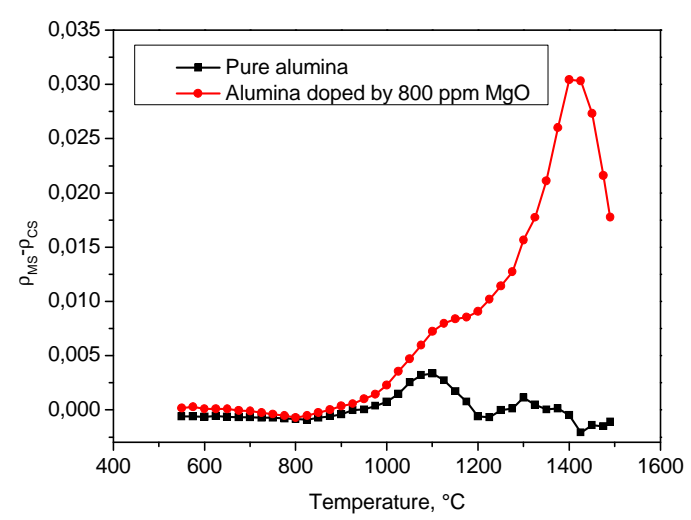

Figure 6: Difference in densities between microwave and conventional sintering as a function of temperature.

\subsection{Thermal gradient evolution during microwave heating}

In this work we refer to the sintering in the microwave cavity as microwave sintering, however we have to take into account that the heating is carried out in presence of both: the microwaves and the susceptor. Therefore, in reality the heating is hybrid, so we have to note that the balance between the contributions of each heating mode may vary as a function of the dielectric parameters of the sample and the susceptor. This may influence the thermal gradient, especially when heating by susceptor is heterogeneous. This effect is seen on each image (see Fig.7 and Fig. 8) in this section. The upper-left side of sample is always hotter than the other parts. 
Temperature distribution and thermal profiles during $\mathrm{MW}$ heating of pure and doped alumina samples are shown respectively in Figures 7 and 8 . A red dashed line marked along the diameter of the sample in Fig. $7 \mathrm{~A}$ and Fig. $8 \mathrm{~A}$, shows where the thermal profiles are measured. The thermal profiles are presented below each image. Selected results represent a temperature distribution at: $850^{\circ} \mathrm{C}, 1050^{\circ} \mathrm{C}, 1250^{\circ} \mathrm{C}$ and $1450^{\circ} \mathrm{C}$.

By analyzing the temperature distribution in the presented figures, we see that in both cases (pure and doped alumina) at $850^{\circ} \mathrm{C}$ (Fig. $7 \mathrm{~A}$ and Fig. $8 \mathrm{~A}$ ) the heating by susceptor is dominant (the edge of the sample has a higher temperature), i.e. the sample is heated mainly in the conventional manner. At $1050^{\circ} \mathrm{C}$ (Fig. $7 \mathrm{~B}$ and Fig. $8 \mathrm{~B}$ ) a beginning of the thermal gradient inversion may be observed. This indicates that the sample efficiently absorbs the microwave energy which is converted into heat. The difference in the temperature between upper-left side and bottom-right side is linked to the heterogeneous heating by the susceptor (as mentioned above) or the heterogeneous distribution of microwaves inside the cavity. From about $1250^{\circ} \mathrm{C}$ (Fig. $7 \mathrm{C}$ and Fig. $8 \mathrm{C}$ ), the thermal gradient inversion is seen. At this temperature microwave heating begins to be the dominant mode of heating. At $1450^{\circ} \mathrm{C}$ gradient inversion is evident and thus is a proof of microwave heating, as shown in Figure $7 \mathrm{D}$ and Figure $8 \mathrm{D}$. The temperature in the center is clearly higher than at the edges of the samples. 

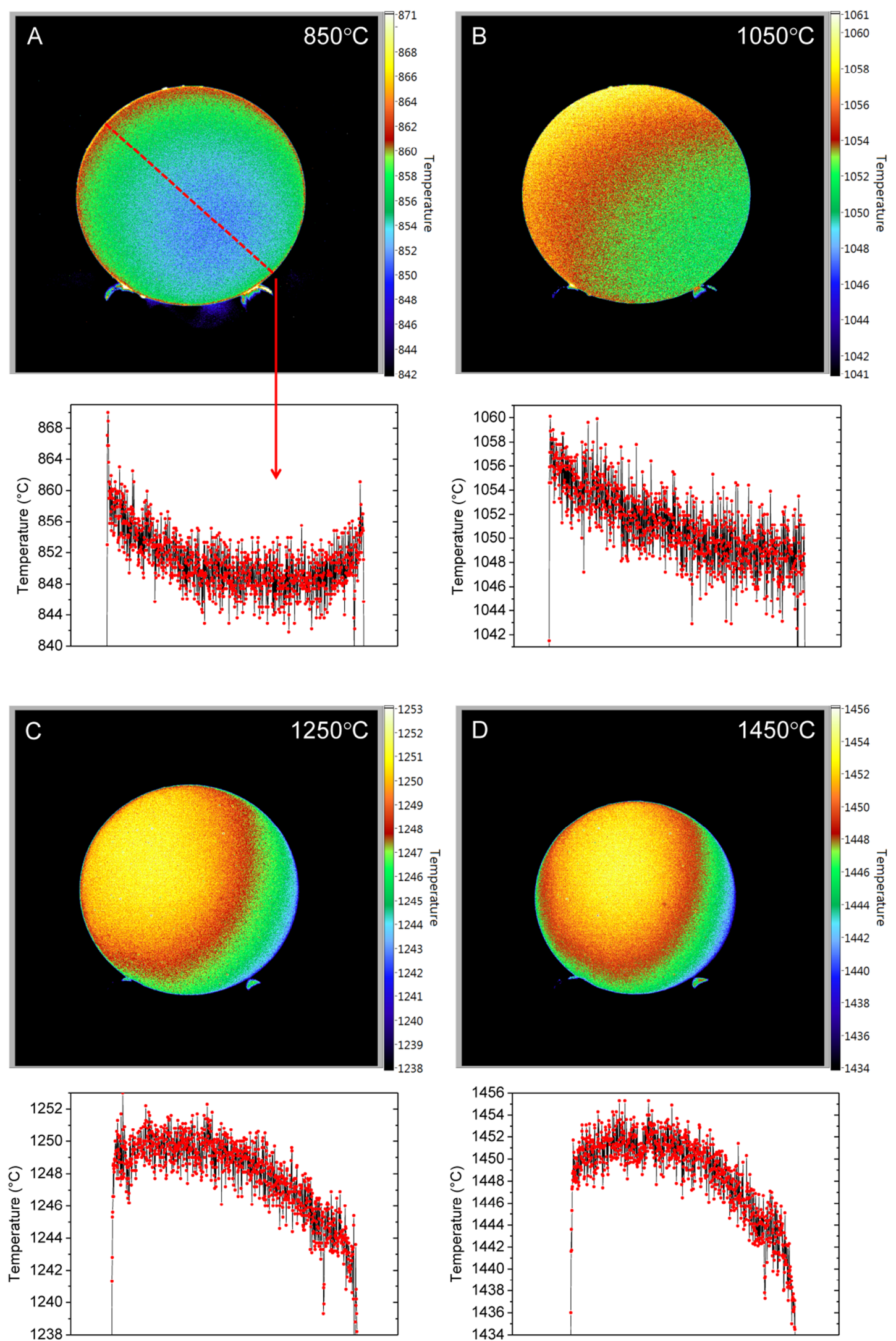

Figure 7: Evolution of the temperature distribution on the surface sample (pure alumina) during sintering in microwave furnace. 

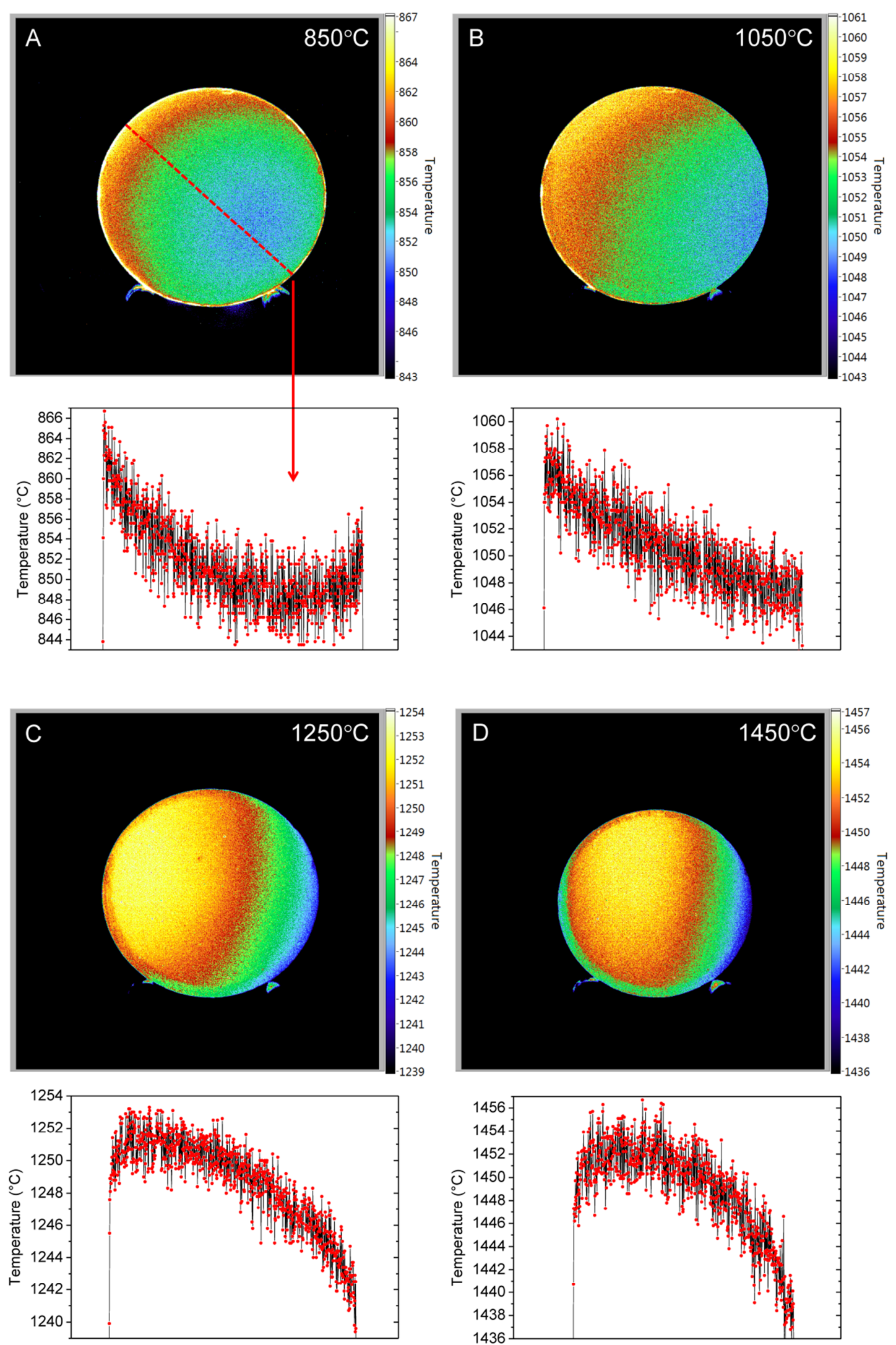

Figure 8: Evolution of the temperature distribution on the surface sample (alumina doped by $800 \mathrm{ppm} \mathrm{MgO)}$ during sintering in microwave furnace. 


\section{Discussion}

In the previous section we reported that in presence of $\mathrm{MgO}$, microwaves enhance the sinterability of alumina when compared to the conventional sintering process. The same effect is not observed in the case of non-doped powder. This fact may indicate that pure alumina does not absorb microwave energy efficiently and in this case the sample is heated mainly by the susceptor. However, the inversion of the thermal gradient is observed for both aluminas (Fig.7 and Fig. 8), i.e. both are efficiently heated by microwaves (above a temperature of about $1000^{\circ} \mathrm{C}$ ). Moreover, the changes in the thermal gradient take place at more or less the same times. So, the fact that, during the sintering of pure alumina, we do not observe the influence of microwaves on the densification process, does not mean that pure alumina does not absorb microwave energy. Based on our observation, we assume that alumina is heated by microwaves (at high temperatures) regardless of MgO dopants. But it is the presence of $\mathrm{MgO}$ that accelerates densification but does not necessarily accelerate the heating on the "global scale".

Soni et al. [12] showed that MgO causes segregation of $\mathrm{Mg}$ and impurities to the grain boundaries and that the concentration of $\mathrm{Mg}$ on grain boundaries is tens of times greater than that within the grains. Hence, we assume that there should be a higher loss factor $\left(\varepsilon^{\prime \prime}\right)$ on grain boundaries than within the grains. In consequence, we can consider a local thermal effect: the temperature at the grain boundaries is enhanced due to a local coupling with microwaves, which results in an accelerated densification. Another hypothesis which should also be taken into account is a nonthermal ponderomotive effect [5]. This effect is more pronounced in materials with more significant microwave absorption i.e. with higher loss factor [6]. So, if for alumina doped by $\mathrm{MgO}$, a loss factor may be higher at grain boundaries than within the grains, this hypothesis may explain observed enhanced densification during sintering in the microwave furnace (Fig. 5 and Fig. 6). 


\section{Conclusion}

A comparative study of microwave and conventional sintering of both pure and doped (800 ppm $\mathrm{MgO}$ ) alumina has shown that microwaves do not influence the densification process of pure alumina. However, when alumina is doped by $\mathrm{MgO}$ a palpable effect is observed, probably due to the higher loss factor on grain boundaries than within the grains, caused by the segregation of $\mathrm{Mg}$ and impurities. Analysis of the thermal gradient evolution showed that both examined powders absorb microwaves and convert them into heat.

During this study we used a homemade optical dilatometer in combination with an original method of measuring temperature distribution. This experimental system seems to be a promising tool in the exploration of the so-called "microwave effect".

\section{Acknowledgements}

The authors gratefully acknowledge Mr. Christophe Meunier, Mr. Bernard Seon and Mr. Bernard Allirand for their help in the realization of this work.

\section{References}

[1] Z. Xie, J. Yang, X. Huang, Y. Huang, Microwave processing and properties of ceramics with different dielectric loss, J. Eur. Ceram. Soc., vol. 19[3] (1999) 381-87.

[2] K. H. Brosnan, G. L. Messing, D. K. Agrawal, Microwave Sintering of Alumina at $2.45 \mathrm{GHz}$, J. Am. Ceram. Soc., vol. 86[8] (2003) 1307-12.

[3] Y. Fang, J. Cheng, D. K. Agrawal, Effect of powder reactivity on microwave sintering of alumina, Materials Letters, vol. 58 [3-4] (2004) 498-501.

[4] J. D. Katz, Microwave Sintering of Ceramics, Ann. Rev. Mater. Sci. 22 (1992) 153-170.

[5] J. H. Booske, R. F. Cooper, S. A. Freeman, K. I. Rybakov, V. E. Semenov, Microwave ponderomotive forces in solid-state ionic plasmas, Phys. Plasmas. 5 (1998) 1664. 
[6] K. I. Rybakov, V. E. Semenov, G. Link, M. Thumm, Preferred orientation of pores in ceramics under heating by a linearly polarized microwave field, J. Appl. Phys. 101 (2007) 084915.

[7] A. Birnboim, J. P. Calame, Y. Carmel, Microfocusing and polarization effects in spherical neck ceramic microstructures during microwave processing, J. Appl. Phys., 85[1] (1999) 478-82.

[8] R. Raj, M. Cologna, J. S. C. Francis, „Influence of Externally Imposed and Internally Generated Electrical Fields on Grain Growth, Diffusional Creep, Sintering and Related Phenomena in Ceramics", J. Am. Ceram. Soc., 94[7] (2011) 1941-65.

[9] E. Pert, Y. Carmel, A. Birnboim, T. Olorunyolemi, D. Gershon, J. Calame, I. K. Lloyd, O. C. Wilson Jr, Temperature Measurements during Microwave Processing: The Significance of Thermocouple Effects, J. Am. Ceram. Soc. 84 [9] (2001) 1981-86.

[10] S. Vaucher, J.-M. Catala-Civera, A. Sarua, J. Pomeroy, M. Kuball, Phase selectivity of microwave heating evidenced by Raman spectroscopy, J. Appl. Phys. 99 (2006) 113505.

[11] D. Żymełka, S. Saunier, J. Molimard, D. Goeuriot, Contactless Monitoring of Shrinkage and Temperature Distribution during Hybrid Microwave Sintering, Adv. Eng. Mater. 13 (2011) 901-905.

[12] K. K. Soni, A. M. Thompson, M. P. Harmer, D. B. Williams, J. M. Chabala, Solute segregation to grain boundaries in MgO doped alumina, Appl. Phys. Lett. 66 (1995) 2795. 


\section{Figure legends}

Figure 1: Microwave heating system.

Figure 2: Dilatometric curve obtained using our optical dilatometer during microwave sintering.

Figure 3: Example of the thermal cycle accomplished in microwave furnace controlled by a homemade computer program.

Figure 4: Comparison of relative densities for pure alumina sintered with constant heating rate $20^{\circ} \mathrm{C} / \mathrm{min}$.

Figure 5: Comparison of relative densities for alumina doped by $800 \mathrm{ppm} \mathrm{MgO}$ and sintered with constant heating rate $20^{\circ} \mathrm{C} / \mathrm{min}$.

Figure 6: Difference in densities between microwave and conventional sintering as a function of temperature.

Figure 7: Evolution of the temperature distribution on the surface sample (pure alumina) during sintering in microwave furnace.

Figure 8: Evolution of the temperature distribution on the surface sample (alumina doped by $800 \mathrm{ppm} \mathrm{MgO}$ ) during sintering in microwave furnace. 


\section{Table legend}

Table 1: Dimensions and densities of green samples 